\title{
Prescription Pattern and Drug Utilization Analysis in Patients with Acute Coronary Syndrome
}

\author{
Dona Saju ${ }^{1, *}$, Cristina Joy ${ }^{1}$, Moushmi Arul Moorthy ${ }^{1}$, Bivin Wilson ${ }^{2}$, Jithin Antony ${ }^{3}$, Sengottuvelu \\ Singaravel ${ }^{4}$, Sheik Haja Sherief ${ }^{4}$, Sivakumar Thangavel ${ }^{5}$
}

1'Department of Pharmacy Practice, Nandha College of Pharmacy, Perundurai Main Road, Erode, Tamil Nadu, INDIA.

${ }^{2}$ Consultant Interventional Cardiologist, G.Kuppuswamy Naidu Memorial Hospital, Papanaickenpalayam, Coimbatore, Tamil Nadu, INDIA.

${ }^{3}$ Department of Clinical Pharmacy, G.Kuppuswamy Naidu Memorial Hospital, Papanaickenpalayam, Coimbatore, Tamil Nadu, INDIA. ${ }^{4}$ Department of Pharmacology, Nandha College of Pharmacy, Perundurai Main Road, Erode, Tamil Nadu, INDIA.

${ }^{5}$ Department of Pharmaceutical Chemistry, Nandha College of Pharmacy, Perundurai Main Road, Erode, Tamil Nadu, INDIA.

\begin{abstract}
Background: Acute Coronary Syndrome (ACS) is one of the major causes of morbidity and mortality in developing countries, which illustrates the need for rational prescribing of drugs to the patients. Aim: The aim of the study is to analyse the prescription pattern and drug utilisation for the drugs prescribed to the patients with ACS by checking the compliance with the standard guidelines provided by the American College of Cardiology/American Heart Association (ACC/AHA). Materials and Methods: A prospective observational study for a period of 6 months was conducted in the cardiology and cardiothoracic departments of a tertiary care hospital. The sample size was determined by using Rao software and percentage of the data was calculated using Microsoft Excel 2007. Results: A total of 270 patients were enrolled in the study in which males (219) dominated females (51) and were found in the age group of 60-69 years (92). Diabetes $(62.9 \%)$ followed by hypertension $(54.8 \%)$ were found to be the dominant risk factors. The prescribing frequency of dual antiplatelet therapy, statins, beta blockers, angiotensin converting enzyme inhibitors/angiotensin receptor blockers and nitrates was 93.3\%, 97.3\%, 94.1\%, $76.3 \% / 14.1 \%$ and $41.2 \%$. The treatment given to the patients was not completely in compliance with the ACC/ AHA guidelines (18.14\%). Conclusion: The study provides an overall insight of the pattern of drugs prescribed to the patients with ACS which reveals the necessity of improving the rational prescribing of drugs in accordance with the standard guidelines.
\end{abstract}

Key words: Acute Coronary Syndrome, Prescription pattern, ACC/AHA guidelines, Rational drug use, Compliance.

\section{INTRODUCTION}

Evidence suggests that Coronary Artery Disease (CAD) has been recognized as the widespread cause of death and disability which is expected to continue increasing in developing countries. CAD develops due to atherosclerosis, leading to an impedance of one or more coronary arteries that supply blood to the heart, which causes an imbalance between myocardial oxygen supply and demand.

CAD can be classified as Chronic Stable Angina and Acute Coronary Syndrome (ACS). Chronic stable angina is described as retrosternal discomfort lasting less than 10 min, brought about or increased with exertion and reduced with rest or nitrates. ACS refers to a spectrum of clinical symptoms varying those for ST-segment elevation myocardial infarction to symptoms found in non-ST-segment elevation myocardial infarction or unstable angina. Unstable Angina is a clinical syndrome caused by partial occlusion of the coronary artery and characterized by ECG ST-segment depression or prominent $T$ wave inversion and no elevation of cardiac biomarkers of necrosis (Troponin T/I or CPK-MB). NSTEMI, also caused by partial occlusion of the coronary artery, is characterized by ECG ST-segment depression or $\mathrm{T}$ wave inversion and positive biomarkers of necrosis, in the
DOI: 10.5530/ijopp.13.1.12

Address for correspondence: Dr. Dona Saju, Pharm.D Intern, Department of Pharmacy Practice, Nandha College of Pharmacy, Erode-638052, Tamil Nadu, INDIA. Phone no: +919207922937 Email Id: donasaju96@gmail. com

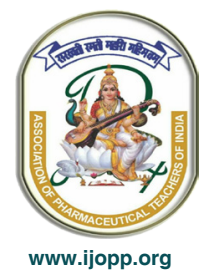


absence of ST-segment elevation. STEMI, on the other hand, is caused by complete occlusion of the coronary artery and characterized by ST-segment elevation, positive cardiac biomarkers or new-onset LBBB due to myocardial necrosis. ${ }^{1}$ The commonly recognised risk factors of ACS are hypertension, diabetes, dyslipidaemia, obesity, smoking and advancing age (males $\geq 35$ years and females $\geq 45$ years).

ACS can be diagnosed by electrocardiography, echocardiography, stress tests, coronary angiography, cardiac biomarkers, serum lipids, electrolytes, blood urea nitrogen and serum creatinine. The goals of treatment after the occurrence of ACS are to bring back the patient into the normal activities and also to use the acute event to re-evaluate the plan of care, particularly lifestyle and risk factor modification. The initial treatment of ACS is achieved by coronary reperfusion using fibrinolysis and/or revascularization using Percutaneous Coronary Intervention (PCI) / Coronary Artery Bypass Graft $(C A B G)$, along with a combination of Anti-ischaemic and Anti-thrombotic agents. Prescribing the medications based on practice guidelines from the American College of Cardiology and American Heart Association (ACC/ AHA) has improved in recent years to promote the proper therapeutic management for secondary prevention of Coronary Artery Diseases. The recommended medications for ACS patients include Statins, Dual Antiplatelet agents, Angiotensin-Converting Enzyme Inhibitors (ACEI) or Angiotensin Receptor Blockers (ARB), Beta-blockers and Nitroglycerin. Treatment administration according to the risk category of the patient is important. Moderate and high-risk patients are admitted to a coronary intensive care unit, depending upon the patients' symptoms and recognized level of risk. High-risk patients should undergo early coronary angiography (within 24-48 hr) and revascularization (with $\mathrm{PCI} / \mathrm{CABG}$ ) if significant coronary artery stenosis is established. Moderate risk patients with positive biochemical markers for infarction typically also undergo angiography and revascularization during hospital admission. $^{2}$

Pharmacists play a key role in addressing many issues that might arise within and during the entire period of therapy. The practice of clinical pharmacy is regarded as an imperative part of a healthcare team to provide the best quality use of medicines. The main role of clinical pharmacists in the management of ACS patients includes the prevention of drug-related problems, management of cardiovascular risk factors and to improve the rational use of drugs. Our intent is to analyse whether the 5 key guidelines recommended medications were prescribed for the ACS patients according to the national practice guidelines from the ACC/AHA and if not, then to find out why they are not prescribing the medications as per the guidelines which is an effort to encourage all the health care providers to improve the quality of patient care.

\section{MATERIALS AND METHODS}

Study design: A prospective observational study was conducted in the Cardiology and Cardiothoracic Departments of a tertiary care hospital for a period of 6 months from February 1, 2018 - June 31, 2018. The study was initiated after receiving the Institutional Ethical Committee approval from the hospital. About 270 patients diagnosed with ACS were analyzed during the study from the time of admission.

\section{Inclusion criteria}

- All patients of either gender with diagnosis confirmed as Acute coronary syndrome

- Patients with a previous history of ACS.

\section{Exclusion criteria}

- Pregnant women

- Out patients

- Patients admitted with other heart diseases (Endocarditis, Rheumatic heart disease, Arrhythmias and Cardiac tumours).

\section{Source of data}

Patient case files (including patient demographics, past medical and medication history, laboratory investigations, drug therapy).

\section{Data collection}

The data was collected from the individual patient case files in a structured proforma. The collected data were documented and evaluated for the study parameters. All 270 patients who met the criteria were followed on a regular basis from the date of admission to the date of discharge. The past medical and medication history of each patient was reviewed. The drugs prescribed to the patients were analysed to evaluate whether the treatment given is in accordance with the guidelines provided by the ACC/AHA. Every modification made on the therapy for the inpatients during the treatment period was updated on the data collection form and reviewed for the reasons of the same.

\section{Statistical analysis}

The sample size was determined using Rao software, Indian Journal of Pharmacy Practice, Vol 13, Issue 1, Jan-Mar, 2020 
where the confidence interval was $95 \%$, an acceptable margin of error was $5 \%$ and the population size was selected as 900 on the basis of ACS patient admissions in the hospital. The percentage of data was calculated using Microsoft Excel 2007 sheet. Chi-squared test was used to compare the rate of recommended drugs prescribed to the patients as per guideline and not as per guideline.

\section{RESULTS}

In the present study, we have randomly collected 270 prescriptions of the inpatients who were admitted with ACS, in which males $(81.1 \%)$ dominated females (18.9\%) as shown in Figure 1. Mean age of the patients was found to be around 59 years, mostly belonging to the age group of $60-69$ years $(34.1 \%)$ followed by $50-59$ years $(26.3 \%)$ and $40-49$ years $(17.8 \%)$ (Table 1$)$.

Diabetes $(62.96 \%)$ constituted the dominant risk factor followed by Hypertension (54.81\%) and Smoking $(35.92 \%)$. Dyslipidaemia (34.07\%), family history of CAD $(14.45 \%)$ and obesity $(4.45 \%)$ were weakly related to ACS risk (Figure 2). The study showed that the predominant symptom of ACS was chest pain contributing to $228(84.45 \%)$ of total cases and the other presented symptoms like profuse sweating, back pain and dyspnoea on exertion contributed to about 112 (41.48\%), $50(18.51 \%)$ and 38 (14.07) patients respectively (Figure 3).

Out of overall patients enrolled in the study, 79 (29.2\%) had Triple vessel disease, which was most common and $45(16.6 \%)$ and $34(12.5 \%)$ had Single vessel disease and Double vessel disease respectively. In our study, 135, 87 and 48 patients were diagnosed with STEMI, NSTEMI and UA respectively, in which 123 patients underwent PCI, 58 patients underwent CABG and 89 patients were under medical therapy only (Table 2).

Among 270 prescriptions analysed, 269(99.6\%) were prescribed with antiplatelets, $200(74.1 \%)$ received anticoagulants, $33(12.2 \%)$ received thrombolytics, $262(97 \%)$ received statins, $254(94.1 \%)$ received betablockers, $112(41.4 \%)$ received nitrates, 36 (13.3\%) were prescribed with calcium channel blockers, 206 (76.2\%) received ACEIs and $38(14.07 \%)$ received ARBs. In the present study, 251(93.3\%) patients received Dual Antiplatelet Therapy (DAPT) while 18(6.6\%) patients received Single Antiplatelet Therapy (SAPT), but 1 patient didn't receive any antiplatelets. We noticed that the utilization rate of statins was high (97.03\%), in which Atorvastatin dominated. Among the betablockers, Metoprolol (37.7\%) was the most prescribed drug followed by Nebivolol (22.5\%) and 100(37\%) patients received Carvedilol, which is an alpha+beta blocker. ACEIs were given to 206(76.3\%) patients in which Ramipril was found to be the most commonly prescribed drug. ARBs were given to 38(14.1\%) patients in which the utilization of Telmisartan dominated in $29(10.7 \%)$ patients. The commonly prescribed nitrates was Isosorbide dinitrate $(28.5 \%)$.

Amlodipine (7.7\%) was the commonly prescribed calcium channel blocker followed by Cilnidipine and Benidipine. In the study, LMWH was prescribed in $71.73 \%$ of patients, among them, $17.03 \%, 52.5 \%$ and $2.2 \%$ received Fondaparinux, Enoxaparin and Dalteparin respectively. UFH was prescribed in $9.2 \%$ of the patients. Oral anticoagulants were prescribed in $3.3 \%$ of the patients. Out of 31 patients who received thrombolytics, $15.5 \%$ were on non-fibrin specific thrombolytic therapy (Streptokinase) and the remaining were on fibrin specific therapy with Tenecteplase $(2.9 \%)$ and Reteplase (4.4\%). Table 3 shows the complete details of the mostly prescribed individual drugs for the patients with ACS.

Out of the 270 prescriptions analysed in the study, about 197 prescriptions complied with the ACC/AHA guideline and the remaining were not in accordance with the guideline (Figure 4). The combination of DAPT, a beta blocker, an ACEI/ARB and a statin is recommended by ACC/AHA guidelines for most patients with ACS unless contraindicated. Our study evaluated the appropriate prescription of cardiac medication upon hospital discharge in patients admitted for ACS and showed that the prescription rate for antiplatelet, beta blocker, ACEI, ARB and statin was 98.5\%, 92.5\%, 65.5\%, 13.3\% and $98.1 \%$ respectively (Figure 5). Chi-squared test is used as a statistical tool to compare the rate of recommended drugs, given to the ACS patients, as per guidelines and not as per guidelines. ' $p$ ' value $<0.05$ is considered as significant. Therefore the value we obtained from Chisquared test is significant at 5\% level (Table 4).

Table 1: Age wise distribution of patients with Acute Coronary Syndrome.

\begin{tabular}{cc} 
Age Groups & $\begin{array}{c}\text { Number of patients } \\
(\boldsymbol{n}=\mathbf{2 7 0})\end{array}$ \\
\hline $20-29$ & 3 \\
$30-39$ & 11 \\
$40-49$ & 48 \\
$50-59$ & 71 \\
$60-69$ & 92 \\
$70-79$ & 36 \\
$80-89$ & 9 \\
\hline
\end{tabular}


Table 2: Pattern of treatment given for different types of ACS.

\begin{tabular}{ccccc} 
Type of & No. of & \multicolumn{3}{c}{ Treatment } \\
\cline { 3 - 5 } ACS & patients & PCI & CABG & $\begin{array}{c}\text { Only medical } \\
\text { therapy }\end{array}$ \\
\hline UA & 48 & 13 & 14 & 21 \\
NSTEMI & 87 & 42 & 16 & 29 \\
STEMI & 135 & 68 & 28 & 39 \\
\hline
\end{tabular}

ACS - Acute Coronary Syndrome

$\mathrm{PCl}$ - Percutaneous Coronary Intervention

CABG - Coronary Artery Bypass Graft

UA - Unstable Angina

NSTEMI - Non-ST-segment Elevation Myocardial Infarction

STEMI-ST-segment Elevation Myocardial Infarction

Table 3: Most frequently prescribed individual drugs in the treatment of ACS.

\begin{tabular}{|c|c|c|}
\hline $\begin{array}{l}\text { Classes of drugs } \\
\text { (No. of patients) }\end{array}$ & Drug name & $\begin{array}{c}\text { No. of } \\
\text { patients }\end{array}$ \\
\hline \multirow{3}{*}{ Anticoagulants } & Enoxaparin & 142 \\
\hline & Fondaparinux & 46 \\
\hline & Heparin & 25 \\
\hline \multirow{4}{*}{ Antiplatelets } & Aspirin & 176 \\
\hline & Clopidogrel & 51 \\
\hline & $\begin{array}{l}\text { Aspirin-Clopidogrel } \\
\text { combination }\end{array}$ & 173 \\
\hline & Ticagrelor & 125 \\
\hline Thrombolytics & Streptokinase & 21 \\
\hline Statins & Atorvastatin & 248 \\
\hline \multirow{2}{*}{ Beta-blockers } & Metoprolol & 102 \\
\hline & Nebivolol & 61 \\
\hline ACEls & Ramipril & 196 \\
\hline \multirow{4}{*}{ Nitrates } & Isosorbide Dinitrate & 77 \\
\hline & Nicorandil & 20 \\
\hline & Isosorbide Mononitrate & 18 \\
\hline & Glyceryl Trinitrate & 12 \\
\hline CCBs & Amlodipine & 21 \\
\hline ARBs & Telmisartan & 29 \\
\hline
\end{tabular}

ACEls - Angiotensin Converting Enzyme Inhibitors

CCBs - Calcium Channel Blockers

ARBs - Angiotensin II Receptor Blockers

\section{DISCUSSION}

In our study, mean age of patients was found to be 59.08 . The patients between ages of 60-69 years constituted the higher number followed by 50-59 years and 40-49 years, which was found to be correlated to the study conducted by Siddaruda Malleshappa Biradar et al. ${ }^{3}$ This shows that risk of ACS is common in older age groups. Out of the 270 patients enrolled in the study, majority
Table 4: Chi-squared test for comparison of rate of recommended drugs prescribed as per guideline and not as per guideline.

\begin{tabular}{cccc} 
Drug Class & $\begin{array}{c}\text { As per ACCIAHA } \\
\text { Guidelines }\end{array}$ & $\begin{array}{c}\text { Not as per ACCI } \\
\text { AHA Guidelines }\end{array}$ & $\mathbf{X}^{2}$ \\
\hline DAPT & $244(90.3 \%)$ & $26(9.6 \%)$ & \\
Statins & $264(97.7 \%)$ & $6(2.2 \%)$ & \\
ACEls/ARBs & $253(93.7 \%)$ & $17(6.2 \%)$ & 133.5 \\
$\begin{array}{c}\text { Beta } \\
\text { Blockers } \\
\text { Nitrates }\end{array}$ & $256(94.8 \%)$ & $14(5.1 \%)$ & \\
\hline
\end{tabular}

$P^{\prime}$ value $<0.05$ is considered as significant. The value of chi-square that we obtained issignificant at the $5 \%$ level.

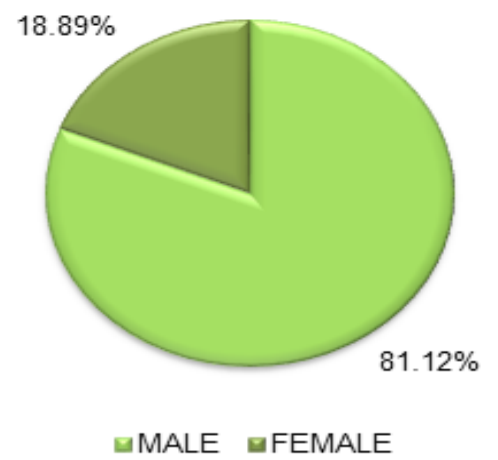

Figure 1: Gender wise distribution of patients with Acute Coronary Syndrome.

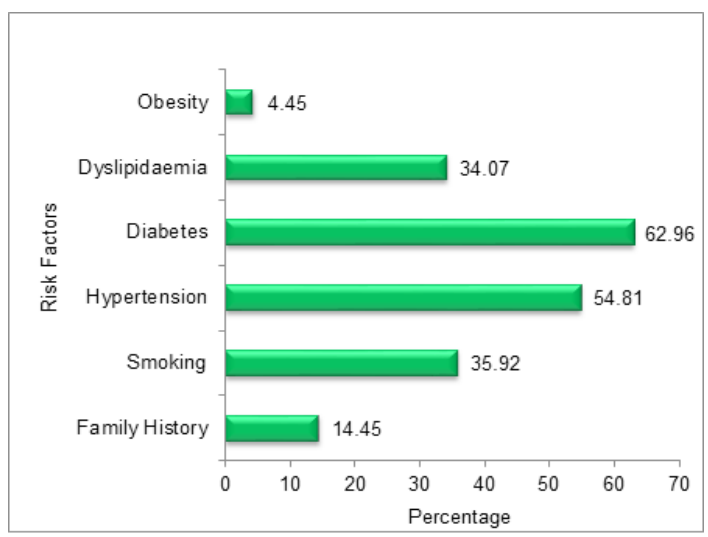

Figure 2: Distribution of selected risk factors in patients with Acute Coronary Syndrome.

were males (219), which parallels the finding of Singh et $a .^{4}$ This shows that the incidence of ACS is higher among the males than females. Some of the risk factors may contribute to the progression of ACS by altering the normal mechanisms of the body. In the study, Diabetes was well found to have an adverse effect on the prognosis of the patients with ACS, whereas, obesity was weakly related to ACS risk, which is in contrast to the study conducted by Avula Naveen et al. ${ }^{5}$ in which obesity Indian Journal of Pharmacy Practice, Vol 13, Issue 1, Jan-Mar, 2020 


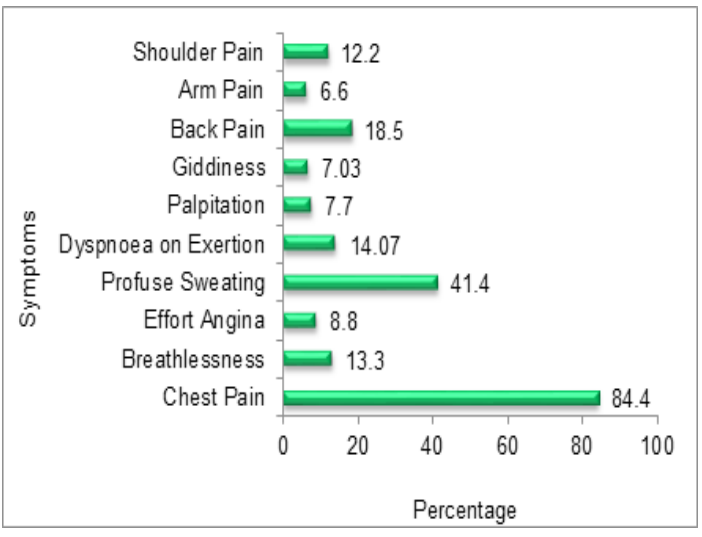

Figure 3: Distribution of symptoms in patients with Acute Coronary Syndrome.

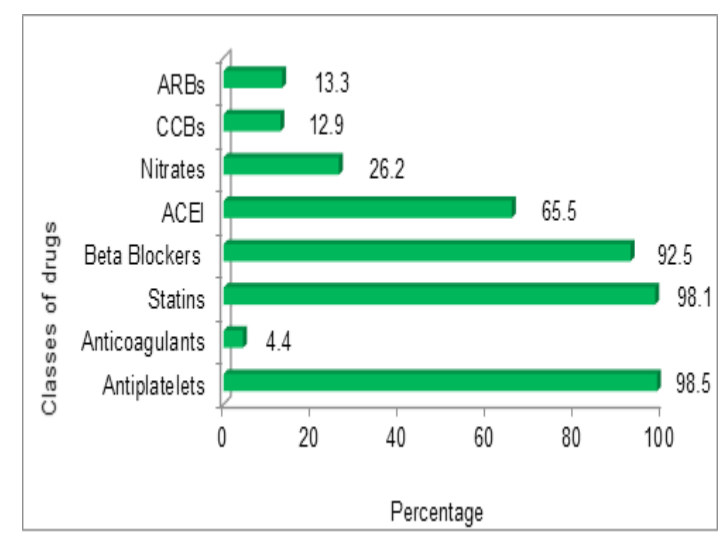

Figure 4: Pattern of discharge medications prescribed to the patients with ACS.

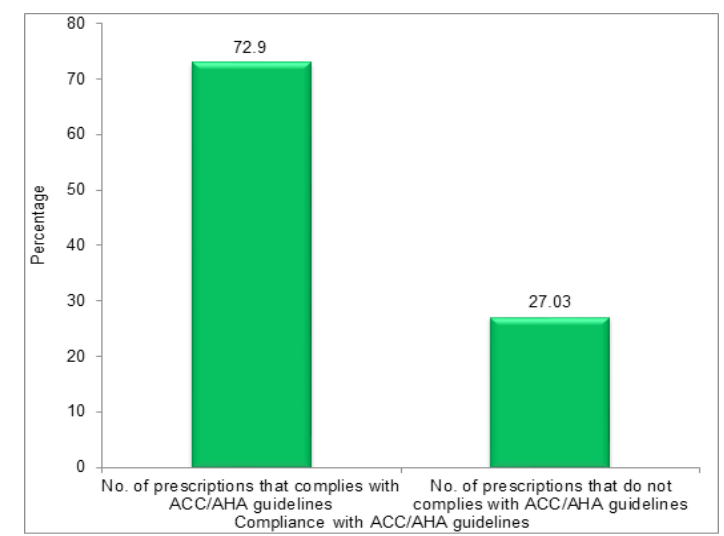

Figure 5: Rate of prescriptions that complies with ACC/AHA Guideline.

constituted the dominant risk factor. In the study, most of the patients were presented with chest pain as the prevailing symptom followed by profuse sweating, as observed by Yadav et al. ${ }^{6}$

Out of overall patients enrolled in the study, majority had Triple vessel disease, while others had Double vessel disease and Single vessel disease, which is in contrast to the study conducted by Mohmad Iqbal Wani et al. ${ }^{7}$ in which Single vessel disease was the most common angiographic finding. In our study, most patients had
Myocardial infarction (NSTEMI and STEMI) and only 48 had UA, as observed by Avula Naveen et al. ${ }^{5}$ In ACS, there is a drift towards early invasive treatment and this is reflected in a marked increase in cardiac care facilities throughout India. If a hospital is equipped with facilities, primary PCI is the treatment of choice for the patients with STEMI. Out of 135 patients diagnosed with STEMI, 68 patients underwent PCI, whereas, 28 patients underwent $C A B G$ and 39 patients were treated only with medical therapy. This is comparable to other studies conducted by Xavier et al. ${ }^{8}$ which show increasing trends of PCI in ACS, indicating early perfusion is a key to the management of patients with STEMI.

Drug utilization research assesses rational drug usage in a better way. ACS treatment involves various categories of drugs - Antiplatelets, Thrombolytics, Anticoagulants, Antihyperlipidemic agents, Antihypertensives, Antacids, Antianginals, Anxiolytics, Analgesics, Antidiabetic agents and antiemetics. These drugs were used to reduce cardiovascular mortality and non-fatal myocardial infarction in ACS. Among 270 prescriptions analysed, most of them were prescribed with Antiplatelets, Anticoagulants, Statins, Beta-blockers, ACEIs and nitrates. Thrombolytics, Calcium channel blockers and ARBs were less commonly prescribed.

The Association of Physicians of India recommends that all patients with MI should receive DAPT. Bleeding risk can be one of the complications for missing one of the antiplatelet therapies. In the present study, most of the patients received DAPT while only $6.6 \%$ of the patients received SAPT. According to meta-analysis by Antithrombotic Trialists Collaboration, use of aspirin in the therapeutic regimen of ACS patients showed a reduction in cardiovascular events with unstable angina by $46 \%$ and also demonstrated that antiplatelet therapy is significantly beneficial in patients with ACS. In the present study, number of patients prescribed with aspirin accounts for about $65.1 \%$, Aspirin-Clopidogrel combination (available as combination in market) was prescribed in $64.07 \%$, Clopidogrel was prescribed in $18.8 \%$ and Ticagrelor in $46.2 \%$ of the patients (Figure 5). In our study, patients were prescribed with Aspirin and Clopidogerl before PCI, whereas after PCI, for the same patients, the drug therapy was changed to Aspirin and Ticagrelor to prevent stenting thrombosis. Also, in DAPT, the drug was given as Aspirin-Clopidogrel combination or Aspirin and Clopidogrel or Aspirin and Ticagrelor. In a study conducted by Tasneem Sandozi et al. ${ }^{9}$ the prescription pattern of various antiplatelet drugs including Aspirin alone was 25.7\% and AspirinClopidogrel combination was $60 \%$, which is similar to our study. 
Treatment with statins in patients stabilized after an ACS lowers the risk of CAD, recurrent $\mathrm{MI}$ and stroke. Among currently available statins, only high dose Atorvastatin $(80 \mathrm{mg}$ ) has been shown to reduce death and ischemic events. The utilization rate of statin was high in our study. This finding correlates with the standard guidelines mentioned for use of the drug in cardiovascular emergencies. The results were found to be similar to various studies conducted by Ian Scott et al. ${ }^{10}$

Beta-blockers are beneficial by attenuating the arrythmogenic potential of damaged myocardium and by reducing myocardial oxygen requirements and thereby, the occurrence of ischemia. In patients with concomitant NSTE-ACS, stabilized heart failure and reduced systolic function, it is recommended to continue beta blocker therapy with one of 3 drugs (Sustained release Metoprolol succinate, Carvedilol or Bisoprolol) to reduce mortality in patients. In our study, about $57.1 \%$ of patients received beta-blockers, mostly Metoprolol. 37\% of patients received alpha+beta-blocker (Carvedilol) and about 5.9\% of patients didn't receive any beta-blockers due to certain contraindications like second or third degree heart block without a cardiac pacemaker and active asthma or reactive airway disease. Similar findings were observed in the studies conducted by Banerjee et al. ${ }^{11}$ in which Metoprolol was the most commonly prescribed beta-blocker.

ACEI is recommended indefinitely in all patients with $\mathrm{LVEF}<40 \%$ and in those with hypertension, diabetes mellitus/stable chronic kidney disease unless contraindicated. ARBs are recommended in patients with heart failure or MI with $\mathrm{LVEF}<40 \%$, who are ACEI intolerant. Our study showed that Ramipril was found to be the most commonly prescribed ACEI and for the patients who are contraindicated to ACEIs, ARBs were given, in which the utilisation of Telmisartan dominated. Further in our study, utilization rate of ACEIs was found to be more than that of beta-blockers. This finding is similar to the study conducted by Martinez et al. ${ }^{12}$

Nitrates dilate normal and atherosclerotic coronary arteries and increase coronary collateral flow. In the present study, the commonly prescribed drug was Isosorbide dinitrate $(28.5 \%)$. But in studies conducted by Abdul Muhit et al..$^{13}$ and Zubair Khalid Labie et al. ${ }^{14}$ Nitroglycerin was most commonly prescribed. All calcium channel blockers cause similar coronary vasodilation and are preferred in vasospastic angina. Verapamil may be beneficial in reducing long term events after AMI. Amlodipine was the commonly prescribed calcium channel blocker. Similar findings were observed with Swathi et al. ${ }^{15}$ The rate of discharge medications for cardiac patients may vary from one study to another. Our study also evaluated the discharge prescriptions of ACS patients which was found to be similar to the study conducted by Lee $\mathrm{et} \mathrm{al.}{ }^{16}$

Out of the 270 ACS patients, about 197 patients were prescribed in accordance with the ACC/AHA guidelines and the remaining was not in accordance with the guideline. The non-compliance were due to certain patient specific conditions, lack of knowledge about the updates of the guidelines, medical recklessness of healthcare professionals and few unspecified reasons. The similar results were observed by Siddharuda Malleshappa Biradar et al. ${ }^{3}$ Significant difference $(p<0.05)$ between the drugs prescribed as per guideline and not as per guideline indicates that there is an increased chance of remission and emergence of adverse outcomes, thus affecting the therapeutic outcome, quality of care and rational use of medicines in patients with Acute Coronary Syndrome.

\section{CONCLUSION}

According to the ACC/AHA guidelines, it is recommended to prescribe Dual Antiplatelet agents, Statins, ACEIs/ ARBs, Beta blockers and Nitrates to the ACS patients during the hospital stay and at the time of discharge, which should be continued for at least 12 months in order to get better outcome. Patient satisfaction can be achieved only if the patient receives rational treatment for the disease. In our study about 270 prescriptions of the ACS patients were analysed, out of which, 197 prescriptions were in accordance with the AAC/AHA guidelines, revealing that the drug therapy is not fully in consistence with the guidelines followed and observes the scope and importance of having a clinical pharmacist to monitor and intervene the drug usage and prescribing patterns.

\section{ACKNOWLEDGEMENT}

We sincerely express our gratitude to the Institutional guide, Principal and H.O.D for giving the internal guidance, encouragement and moral support throughout the study. We also thank our Hospital guide and Project co-guide who responded promptly and provided clinical guidance for the accomplishment of the study.

\section{CONFLICT OF INTEREST}

The authors declare no conflict of interest.

\section{ABBREVIATIONS}

ACS: Acute Coronary Syndrome; ACC/AHA: American College of Cardiology/American Heart Association; CAD: Coronary Artery Disease; NSTEMI: Non-STsegment Elevation Myocardial Infarction; STEMI: STsegment Elevation Myocardial Infarction; UA: Unstable Angina; PCI: Percutaneous Coronary Intervention; 
CABG: Coronary Artery Bypass Graft; DAPT: Dual Antiplatelet Therapy; SAPT: Single Antiplatelet Therapy;

ACEI: Angiotensin Converting Enzyme Inhibitor; ARB: Angiotensin Receptor Blocker.

\section{REFERENCES}

1. Talwar KK, Yash PS, Thakur JS, Rajiv M, Shiv B. Clinical Management Guidelines for Coronary Artery Disease for National Programme and Control of Diabetes, Cardiovascular Disease and Stroke. Government of India-WHO; Collaborative Programme 2008-2009. 2017.

2. Joseph TD, Robert LT, Gry CY, Gary TM, Barbara GW, Micheal PL. Pharmacoherapy: A Pathophysiological Approach. 6th Edition. New York. McGraw Hill, Medical Publishing Division. 2014;291-315.

3. Siddaruda MB, Pournamy, Neeraja N, Pathi I, Manjunatha RG, Vijayakumar W. Prescribing Pattern, Drug Utilization and Clinical Pharmacy Services in Acute Coronary Syndrome patients. American Journal of Drug Discovery and Development. 2017;7(2):63-9.

4. Singh PS, Singh G, Singh SK. Clinical profile and risk factors in acute coronary syndrome. Chest. 2013;180:90.

5. Naveen A, Sravani MR, Venkat JN. Drug utilization patterns in acute coronary syndrome at a tertiary care teaching hospital: A retrospective, non-interventional and observational study. The Pharma Innovation. 2017;6(9, Part H):538.

6. Yadav P, Joseph D, Joshi P, Sakhi P, Jha RK, Gupta J. Clinical profile and risk factors in acute coronary syndrome. National J Comm Med. 2010;1(2):150-1.

7. Wani MI, Rashid A, Beig JR, Ajaz S. Acute Coronary Syndrome (ACS) in the Young: Angiographic Features and Risk Factor Analysis of Patients with
ACS before the Age of 35 Years. International Journal of Scientific Study. 2017;5(4):244-8.

8. Xavier D, Pais P, Devereaux PJ, Xie C, Prabhakaran D, Reddy KS, et al. Treatment and outcomes of acute coronary syndromes in India (CREATE): A prospective analysis of registry data. The Lancet. 2008;371(9622):1435-42.

9. Sandozi T, Nausheen F. Drug utilization study in ischemic heart diseases associated with diabetes and hypertension. Int J Pharma Bio Sci. 2010;1(3).

10. Scott IA, Heath K, Harper C, Clough A. An Australian comparison of specialist care of acute myocardial infarction. International Journal for Quality in Health Care. 2003;15(2):155-61.

11. Banerjee S, Kumar V, Ramachandran P, Kamath A. Does the pharmacological management of unstable angina vary with age and gender-a descriptive study. Journal of Clinical and Diagnostic Research. 2010;4:3150-7.

12. Martinez M, Agusti A, Arnau JM, Vidal X, Laporte JR. Trends of prescribing patterns for the secondary prevention of myocardial infarction over a 13-year period. European Journal of Clinical Pharmacology. 1998;54(3):203-8.

13. Muhit MA, Rahman MO, Raihan SZ, Asaduzzaman M, Akbar MA, Sharmin N, et al. Cardiovascular disease prevalence and prescription patterns at a tertiary level hospital in Bangladesh. Journal of Applied Pharmaceutical Science. 2012;2(3):8.

14. Khalid LZ, Sultana R, Bake A, Sikder K, Jahan K. Surveillance on prescribed cardiovascular drugs by generic names in Dhaka city of Bangladesh. International Journal of Pharmacy and Life Sciences. 2013;4(4).

15. Swathi M, Akhilendran R, Hima M, Fahida F, Veena V, Pradeep P. Analysis of drug prescribing pattern and sociodemographic background in patients with Coronary Artery Disease in a tertiary care hospital. EJPMR. 2016;3(11):352-8.

16. Lee CW. Dual antiplatelet therapy for coronary artery disease. Circulation Journal. 2014:CJ-14. 